\title{
Long-term exposure to gefitinib induces acquired resistance through DNA methylation changes in the EGFR-mutant PC9 lung cancer cell line
}

\author{
HIDEKI TERAI $^{1}$, KENZO SOEJIMA ${ }^{1}$, HIROYUKI YASUDA ${ }^{1}$, TAKASHI SATO ${ }^{1}$, KATSUHIKO NAOKI $^{2}$, \\ SHINNOSUKE IKEMURA ${ }^{1}$, DAISUKE ARAI ${ }^{1}$, KEIKO OHGINO ${ }^{1}$, KOTA ISHIOKA ${ }^{1}$, \\ JUNKO HAMAMOTO $^{1}$, YAE KANAI ${ }^{3}$ and TOMOKO BETSUYAKU ${ }^{1}$ \\ ${ }^{1}$ Division of Pulmonary Medicine, Department of Internal Medicine and ${ }^{2}$ Keio Cancer Center, School of Medicine, \\ Keio University; ${ }^{3}$ Division of Molecular Pathology, National Cancer Center Research Institute, Tokyo, Japan
}

Received September 9, 2014; Accepted October 13, 2014

DOI: 10.3892/ijo.2014.2733

\begin{abstract}
This study was designed to identify epigenetically regulated genes and to clarify the contribution of epigenetic alteration to acquired resistance to epidermal growth factor receptor-tyrosine kinase inhibitors (EGFR-TKIs). We established a gefitinib-resistant lung cancer cell line, PC9, which was originally gefitinib-sensitive, by serial long-term exposure to gefitinib. RNA and DNA were collected from both gefitinib-sensitive and -resistant PC9 cells, and comprehensive DNA methylation and mRNA expression analyses were performed using Infinium HumanMethylation27 Bead Arrays and Agilent SurePrint G3 Human Gene Expression $8 \times 60 \mathrm{~K}$ Array, respectively. DNA methylation was increased in 640 genes in gefitinib-resistant cells compared to parental cells. Among them, we selected 29 candidate genes that presented a decrease in mRNA expression in resistant PC9. We further studied four of the selected genes (C10orf116, IGFBP3, KL, and $S 100 P$ ) and found that $K L$ or $S 100 P$ silencing by siRNA induced a decrease in gefitinib sensitivity compared to that in the negative control in PC9. In conclusion, $K L$ and S100P could be potential targets to overcome resistance to EGFR-TKIs.
\end{abstract}

\section{Introduction}

Lung cancer is the leading cause of cancer-related deaths worldwide. Non-small cell lung cancer (NSCLC) accounts for $\sim 85 \%$ of lung cancers (1). The median survival time of patients with advanced NSCLC is $<1$ year (2).

Correspondence to: Dr Kenzo Soejima, Division of Pulmonary Medicine, Department of Internal Medicine, School of Medicine, Keio University, 35 Shinanomachi, Shinjuku-ku, Tokyo, Japan E-mail: ksoejima@cpnet.med.keio.ac.jp

Key words: epidermal growth factor receptor, EGFR-TKI, drug resistance, DNA methylation, KL, S100P
Epidermal growth factor receptor (EGFR)-targeted therapy, using epidermal growth factor receptor-tyrosine kinase inhibitors (EGFR-TKIs), for patients harboring EGFR mutations such as exon 19 deletions or the L858R point mutation in exon 21, is one of the most accepted and well-studied molecular targeted therapies to date (3-5). Despite a high proportion of response to EGFR-TKIs, many patients with EGFR mutations will relapse and eventually develop resistance to EGFR-TKIs. Recently, several studies have already elucidated the mechanism underlying acquired resistance to EGFR-TKIs in NSCLC harboring EGFR mutations. We have previously reported the role of FGF2-FGFR1 activation as one of the mechanisms of acquired resistance (6). However, the mechanisms of acquired resistance are not yet fully understood.

Epigenetic modifications play an important role in the control of gene expression in mammalian cells. Recently, aberrant DNA hypermethylation of $\mathrm{CpG}$ islands in the gene promoter region has become one of the major mechanisms for silencing tumor suppressor or other cancer-associated genes such as O6-methylguanine-DNA methyltransferase $(M G M T)$, $p 16, R A R b, T I M P-3$, and $D A P K$ in lung cancer cells $(7,8)$.

Furthermore, DNA hypermethylation is often associated with responses to chemotherapy (9). One classical example is that $M G M T$ promoter methylation in gliomas is a useful predictor of tumor responsiveness to the alkylating agent carmustine as well as a predictor of overall and disease-free survival in gliomas (10).

In addition, drug-induced DNA hypermethylation could be a mechanism that modifies tumor cell response to chemotherapeutic agents (11-15). Therefore, one possible reason for the development of chemoresistance in NSCLC might be the epigenetic inactivation of certain tumor suppressor genes due to chemotherapy treatment. Since most studies have focused on a limited number of candidate genes, many epigenetically silenced tumor suppressor genes involved in cancer-chemoresistance remain unidentified $(12,14)$. Microarray-based screen pairing of the differential genetic profile of chemosensitive and chemoresistant cell lines have been reported (16-20). The association between PTEN DNA methylation and acquired 
resistance to gefitinib in NSCLC cells was previously reported (21). However, only few reports focused on the relationship between EGFR-TKI resistance and aberrant DNA methylation $(22,23)$. Furthermore, DNA demethylating agent, 5-azacytidine, might increase the cellular sensitivity to gefitinib and control NSCLC cell growth and apoptosis (24).

In this study, the cells described in a previous report (6) were used. PC9 gefitinib-resistant (PC9 GR), gr1, and gr3 cells acquired resistance to gefitinib through FGF2-FGFR1 activation. We further sought to clarify the additional mechanisms involved in gefitinib resistance, beside FGF2-FGFR1 activation.

To elucidate the key epigenetic regulation mechanisms responsible for gefitinib resistance, the global DNA methylation patterns of gefitinib-sensitive and -resistant lung cancer cell lines were compared.

\section{Materials and methods}

Cell lines. The human NSCLC cell line PC9 [EGFR exon 19 deletion (delE746-A750)] was used. PC9 was kindly gifted by S. Kobayashi (Beth Israel Deaconess Medical Center, Boston, MA, USA) (25). Cells were cultured in RPMI-1640 growth medium, supplemented with $10 \%$ fetal bovine serum (FBS) at $37^{\circ} \mathrm{C}$ in a humidified $5 \% \mathrm{CO}_{2}$ incubator.

Establishment of PC9 GR, grl, and gr3 cells. We established gefitinib-resistant cell lines by long-term exposure to gefitinib as characterized previously (6). FGFR1 and FGF2 expression was increased in PC9 GR cells compared to that in PC9 naïve (PC9 na) cells. Gefitinib-resistant clones were also established from PC9 GR cells, namely, PC9 GR1 and gr3. Proliferation of these PC9 GR cells (PC9 GR, gr1, and gr3) was partly dependent on the activation of the FGF2-FGFR1 pathway.

Reagents. The cell lines were treated with the following inhibitors as single agents at various concentrations. Gefitinib was a gift from AstraZeneca Pharmaceuticals (London, UK). 5-Azacitidine was purchased from Wako Pure Chemical Industries (Osaka, Japan).

Cell proliferation assay. The MTS assay was performed according to the manufacturer's protocol by using CellTiter 96 AQueous One Solution Assay (no. G3582; Promega Corporation, Madison, WI, USA). Briefly, $5 \times 10^{2}$ cells were seeded per well in 96-well plates and allowed to attach for $24 \mathrm{~h}$. The cells were then treated with gefitinib at various concentrations. Control cells were treated with the same concentration of the vehicle dimethyl sulfoxide (DMSO). Forty-eight or $72 \mathrm{~h}$ after treatment with the drugs, cell growth was analyzed.

Microarray analysis. Agilent SurePrint G3 Human Gene Expression 8x60K Array (G4851A; Agilent Technologies, Inc., Santa Clara, CA, USA) was used to monitor the expression profiles of the samples. Total RNA was prepared using the RNeasy Mini kit (no. 74106; Qiagen, Hilden, Germany), and labeled cRNA was prepared using standard Agilent protocols. The $\log _{2}$ of the fold change to PC9 na was calculated for each sample by using GeneSpring GX software (Agilent Technologies, Inc.). These microarray data were deposited in the Gene Expression Omnibus (GEO) database under dataset accession no. GSE38302.

Infinium assay. Genomic DNA was extracted from cell lines by using a DNeasy Blood \& Tissue kit (Qiagen). Aliquots (500 ng) of DNA were subjected to bisulfite conversion by using an EZ DNA Methylation-Gold kit (Zymo Research, Irvine, CA, USA). DNA methylation status of $27,578 \mathrm{CpG}$ loci was examined at a single-CpG resolution by using the Infinium HumanMethylation27 Bead Arrays (Illumina, San Diego, CA, USA). After hybridization, the specifically hybridized DNA was fluorescence-labeled by a single-base extension reaction and detected using a BeadScan reader (Illumina), in accordance with the manufacturer's protocols. The data were then assembled using GenomeStudio methylation software (Illumina). At each $\mathrm{CpG}$ site, the ratio of the fluorescence signal was measured using a methylated probe relative to the sum of the methylated and unmethylated probes, i.e., the so-called $\beta$-value, which ranges from 0.00 to 1.00 , reflecting the methylation level of an individual $\mathrm{CpG}$ site.

Quantitative RT-PCR. Total cellular RNA was prepared from the cells by using an RNeasy Mini kit, and $1.0 \mu \mathrm{g}$ of the RNA was then reverse transcribed to cDNA by using TaqMan Reverse Transcription Reagents (N8080234; Invitrogen Life Technologies, Carlsbad, CA, USA). For quantitative reverse transcription-polymerase chain reaction (RT-PCR) analysis, we used an ABI Prism 7000 Sequence Detection System (Invitrogen Life Technologies). Human glyceraldehyde 3-phosphate dehydrogenase $(G A P D H)$ was used for normalization of input cDNA. The probe ID and primer sequences are available on request.

5-aza-dC treatment. PC9 cells were seeded at a density of $1.0 \times 10^{5}$ cells/well in 6 -well plates on day 0 and then allowed to attach for a 24-h period. 5-Aza-2'-deoxycytidine (5-aza-dC) was then added at a final concentration of $1 \mu \mathrm{M}$. After $48 \mathrm{~h}$, the medium was changed, and 5-aza-dC was added again at the same final concentration. Total RNA was extracted from all cells on day 5 .

KL and S100P silencing by siRNAs. PC9 na cells were transfected with a final concentration of $20 \mathrm{nM}$ of S100P siRNA, KL siRNA, or negative control siRNA (no. s194780 for S100P siRNA and no. s225120 for KL siRNA; Ambion, Grand Island, NY, USA). Silencer Select and negative control mix Silencer Select (Ambion) were used according to the manufacturer's instructions. For transfection, siLentFect (no. 170-3361; Bio-Rad, Hercules, CA, USA) was used according to the manufacturer's protocol. Knockdown of $K L$ and $S 100 P$ expression was confirmed using quantitative RT-PCR. For the viability assay, cells were seeded in 6-well plates at a density of 100,000 cells/well $24 \mathrm{~h}$ after transfection with S100P siRNA, KL siRNA, or negative siRNA control. The following day, the cells transfected with siRNAs were seeded in 96-well plates at a density of 500 cells/well and were incubated with four different doses of gefitinib for an additional $72 \mathrm{~h}$. Cell viability 
A DNA methylation increased Total 1786 genes

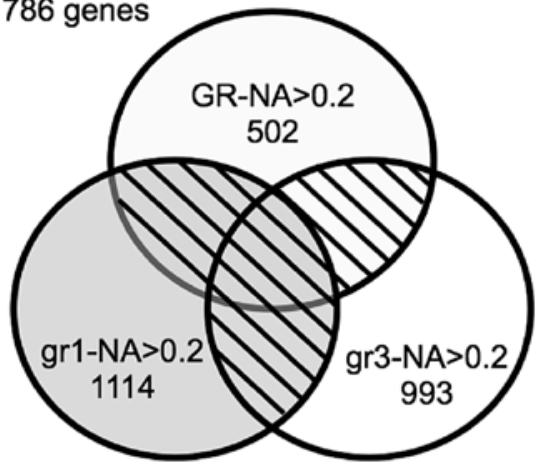

640 genes (at least 2 groups, shaded area)

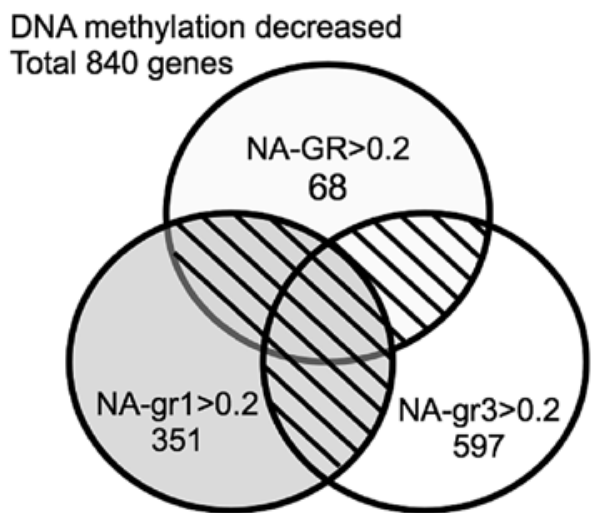

152 genes (at least 2 groups, shaded area)

B

\begin{tabular}{|l|l|l|}
\hline \multicolumn{2}{|l|}{} & Methylation change \\
\cline { 2 - 3 } \multicolumn{2}{|l|}{} & RE-NA>0.2 \\
\hline Relative change of & Down & ALDH3A1, C10orf116 (APM2), FGFR3, GDA, GDPD3, \\
mRNA expression & $(<-2)$ & HSD17B2, IGFBP3, KL, KRT13, KRT5, LY6D, \\
& & METTL7A, MFAP5, MGP, MORC1, NCCRP1, PRR15L, \\
& PRSS2, S100P, SDCBP2, SKAP1, SLC22A18, SPNS3, \\
& SPRR3, TFF2, TIRAP, UBXN10, VCY, VSIG2 \\
\hline
\end{tabular}

Figure 1. Comprehensive analysis of the DNA methylation status and mRNA expression. (A) The number of genes identified by Infinium assay is shown. We identified multiple methylation changes in PC9 gefitinib-resistant (PC9 GR) cell lines (PC9 GR, gr1 and gr3) compared to PC9 naïve (PC9 na) cells. In total, 1,786 genes were hypermethylated on $\mathrm{CpG}$ sites (left), while only 840 genes became hypomethylated following exposure to gefitinib (right). DNA methylation increased in 640 genes in at least two gefitinib-resistant cell lines compared to PC9 na cells (left, shaded area), while 152 genes were hypomethylated in at least two gefitinib-resistant cell lines (right, shaded area) compared to PC9 na cells. (B) List of genes grouped by change in mRNA expression and DNA methylation. Twenty-nine genes showed decreased mRNA expression and increased DNA methylation.

was measured using the MTS assay. Cells treated with DMSO were used as the control.

\section{Results}

Analysis of DNA methylation changes associated with gefitinib resistance by Infinium assay. To identify DNA methylation changes associated with differential gefitinib sensitivity, we used PC9 na and isogenic gefitinib-resistant cell lines, PC9 GR, gr1, and gr3. We performed genome-wide DNA methylation profiling of PC9 GR, gr1, gr3, and PC9 na by using Infinium HumanMethylation27 Bead Arrays that comprise 27,578 CpG sites across $>14,000$ genes.

First, differentially CpG-methylated genes were extracted based on a significant increase or decrease of their beta score between PC9 GR, gr1, or gr3 and PC9 na. Following exposure to gefitinib, we identified 1,786 genes, which were hypermethylated in at least one of the PC9 GR cell lines compared to PC9 na cells, while only 840 genes were hypomethylated in at least one of the resistant cell lines compared with PC9 na cells, suggesting that hypermethylation occurs more frequently than hypomethylation during the selection process for acquired gefitinib resistance (Fig. 1A).

DNA methylation increased in 640 genes in at least two of the three gefitinib-resistant cell lines compared to PC9 na cells (Fig. 1A, left, shaded area). In contrast, DNA methylation decreased in 152 genes in at least two of the three gefitinib-resistant cell lines compared to PC9 na cells (Fig. 1A, right, shaded area).
Association of DNA methylation and gene expression. In a second step, mRNA expression profiles of sensitive PC9 na cells and PC9 GR cell lines were analyzed using Agilent SurePrint G3 Human Gene Expression 8x60K Array. For each $\mathrm{CpG}$ on the Infinium HumanMethylation27 Bead Arrays, the corresponding gene was matched to the Agilent cDNA Microarray data by using the Entrez Gene ID. Signal intensity in the gene expression data was used as an indicator of the expression level.

We further screened for the genes of interest by identifying those that presented a decreased mRNA expression in at least two of the three resistant cell lines.

Finally, we compared our candidate genes to those reported by other investigators to be downregulated through DNA methylation in chemoresistant cell lines $(15,19,26)$. This approach allowed us to identify 29 genes that were downregulated in PC9 GR cell lines and presented a change in DNA methylation (Fig. 1B). Genes with increased CpG methylation and concomitant decreased gene expression included ALDH3A1, C10orf116 (APM2), FGFR3, GDA, GDPD3, HSD17B2, IGFBP3, KL, KRT13, KRT5, LY6D, METTL7A, MFAP5, MGP, MORC1, NCCRP1, PRR15L, PRSS2, S100P, SDCBP2, SKAP1, SLC22A18, SPNS3, SPRR3, TFF2, TIRAP, $U B X N 10, V C Y$, and VSIG2.

In this study, we focused on four genes (C10orf116, $I G F B P 3, K L$, and $S 100 P$ ) based on the expression pattern and previous reports, for further examination. The methylation change and expression of mRNA from the array data are presented in Fig. 2. 
A

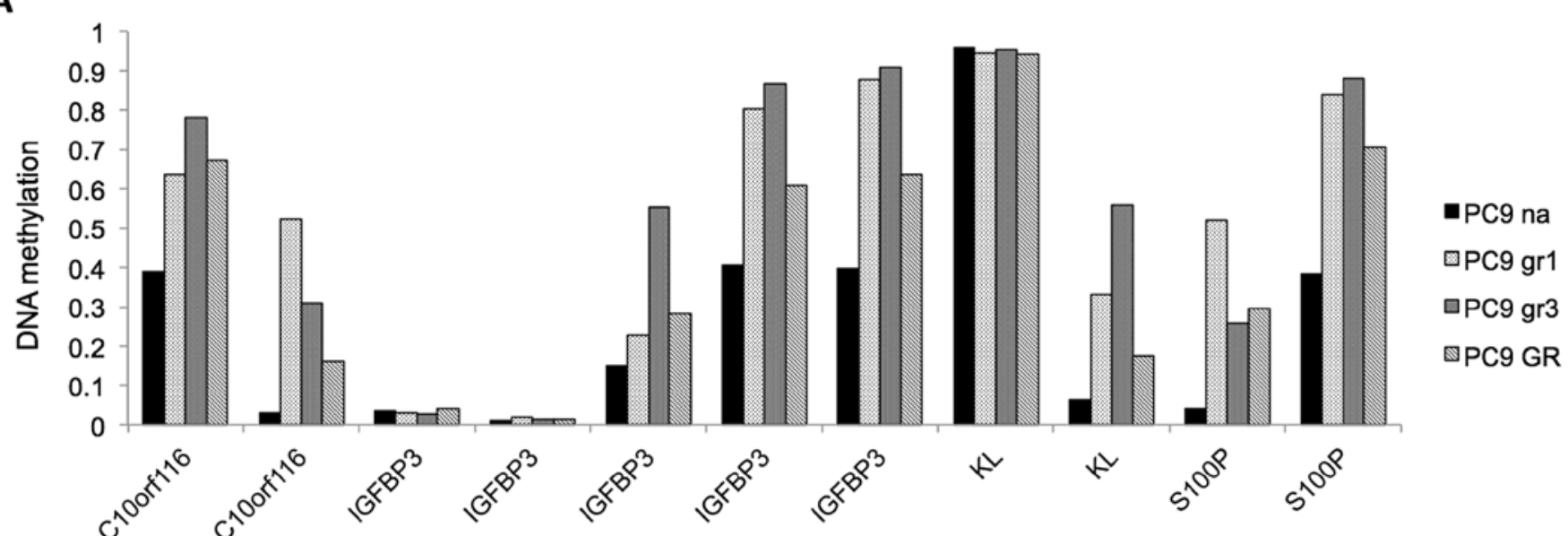

B

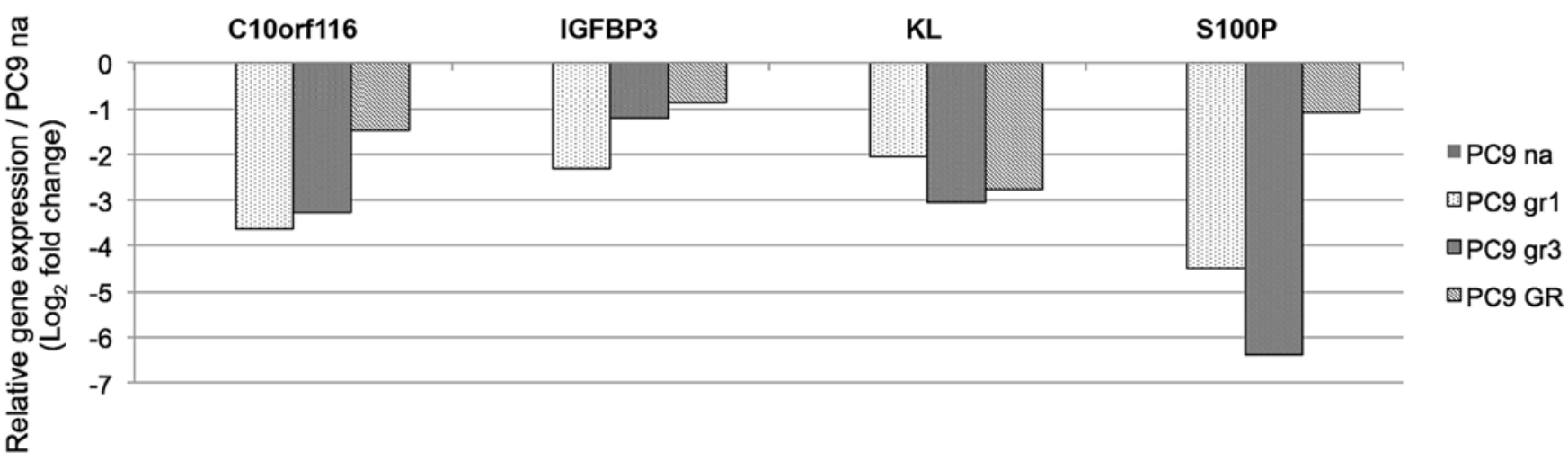

Figure 2. DNA methylation status and mRNA expression of candidate genes in each cell line. (A) Beta scores of selected genes in each cell line as determined by Infinium HumanMethylation27 Bead Arrays. Four genes that were hypermethylated in PC9 gefitinib-resistant (PC9 GR) cells compared to gefitinib sensitive parental cells [PC9 naïve (PC9 na)] were selected. (B) mRNA expression of the selected genes in each cell line as determined by an Agilent SurePrint G3 Human Gene Expression 8x60K Array. In resistant cell lines, the mRNA expression of the selected genes was decreased compared with that in PC9 na cells.

Confirmation of $m R N A$ expression by quantitative RT-PCR. Quantitative RT-PCR confirmed the observed microarray expression changes in the selected four genes $(K L, S 100 P$, C10orf116 and IGFBP3) (Fig. 3). These data indicated that the expression of the four genes was decreased in PC9 resistant cells.

Re-expression of target genes by 5-aza-dC treatment. To make sure that the gene expression of the four genes was regulated by DNA methylation, we performed DNA demethylating experiments. Treatment with 5-aza-dC of PC9 GR cells caused the re-expression of two genes (KL and S100P) (Fig. 4). These findings indicated that $S 100 P$ and $K L$ were in part epigenetically silenced by methylation of $\mathrm{CpG}$ sites during gefitinib exposure.

Acquisition of gefitinib resistance after siRNA-mediated knockdown of KL and S100P in PC9 na cells. To determine whether decreased expression of $K L$ and S100P affects the sensitivity of PC9 cells to gefitinib, we performed gene knockdown experiments by using siRNA. Knockdown of $K L$ and $S 100 P$ were confirmed by quantitative RT-PCR (Fig. 5A).
As expected, $K L$ or $S 100 P$ knockdown resulted in a partial gain of gefitinib resistance in PC9 na cells (Fig. 5B).

Taken together, these results demonstrated that the loss of $K L$ and $S 100 P$ expression is involved in the acquired gefitinib resistance of PC9 na cells.

\section{Discussion}

Previously, we demonstrated that the main mechanism underlying acquired resistance to gefitinib in the resistant cells PC9 GR, grl, and gr3 involved the activation of the FGF2-FGFR1 pathway (6). However, the inhibition of FGF2-FGFR1 pathway by a FGFR inhibitor or siRNA could not fully recover the sensitivity to gefitinib in the resistant cells. Thus, we hypothesized that other mechanisms may be involved in the acquired resistance to gefitinib. In this study, we focused on epigenetics as a potential mechanism.

Epigenetics is defined as heritable genetic changes, which do not change the DNA sequence itself (26). These changes include DNA methylations and chromatin modifications. Epigenetics regulate multiple gene expression and play multiple important roles in various biological processes, 


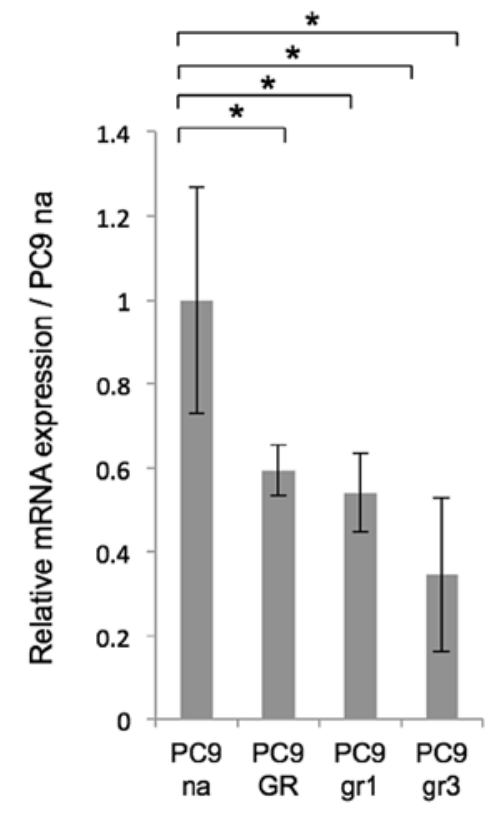

c10orf116
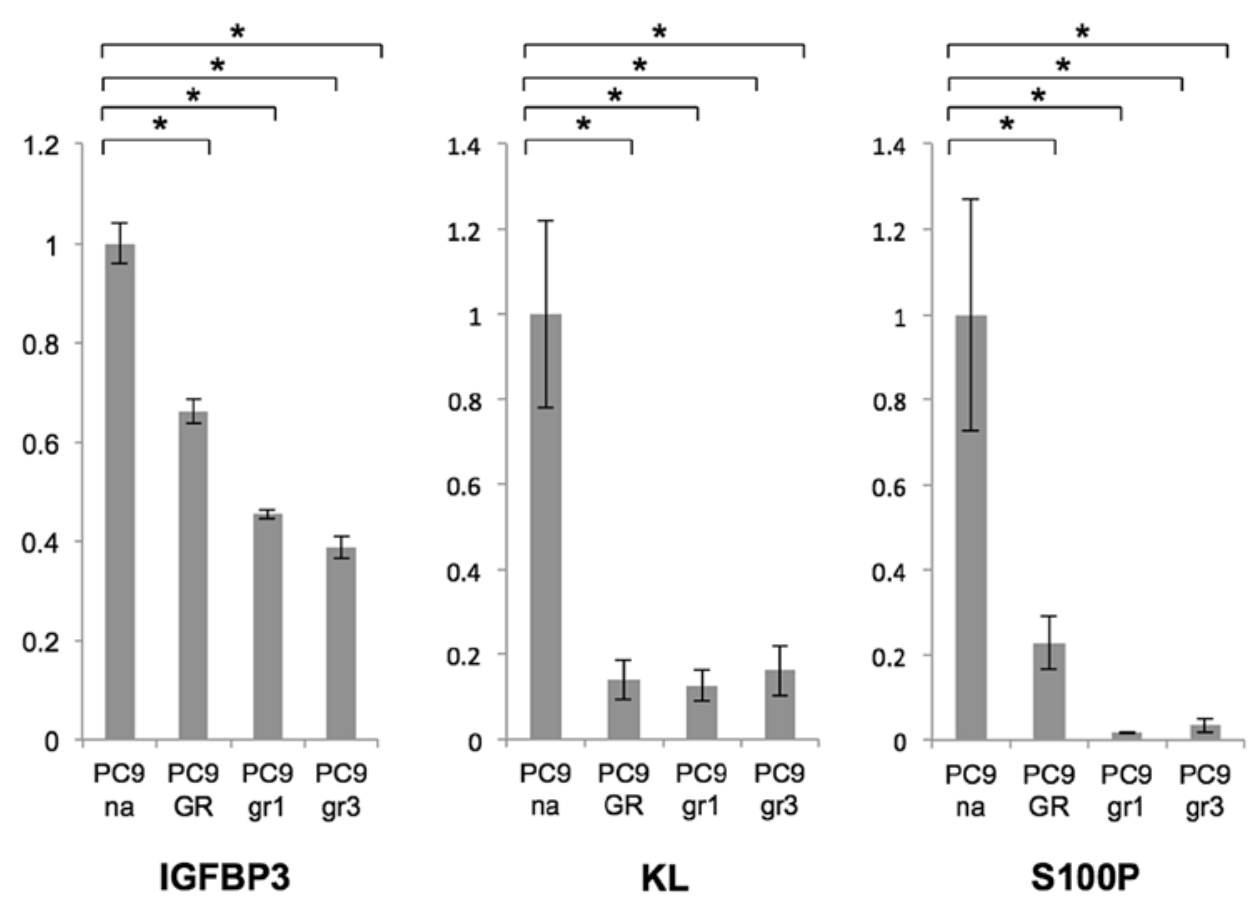

Figure 3. Confirmation of candidate gene mRNA expression. Candidate gene mRNA expression was analyzed by quantitative reverse transcription-polymerase chain reaction (RT-PCR) by using total RNA extracted from PC9 naïve (PC9 na), PC9 gefitinib-resistant (PC9 GR), gr1, and gr3 cells. Quantitative RT-PCR confirmed the low expression level of $C 10$ orf116, IGFBP3, KL, and S100P in PC9 GR, gr1, and gr3 cells. Each bar represents the mean $\pm \mathrm{SD}$. $\mathrm{P}<0.05$, Student's t-tests.

KL

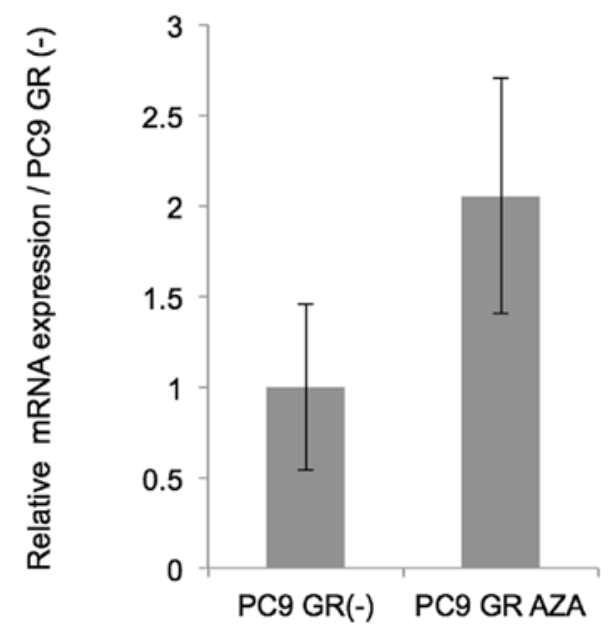

S100P

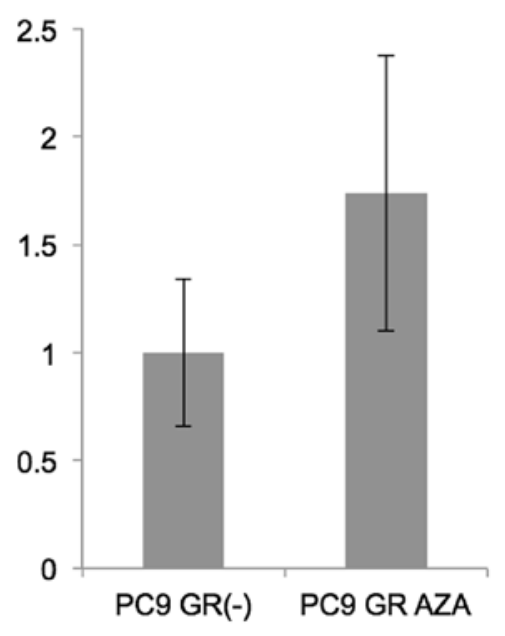

Figure 4. Re-expression of candidate genes in cell lines treated with a demethylating agent. Candidate gene mRNA expression was analyzed by quantitative reverse transcription-polymerase chain reaction (RT-PCR) by using total RNA extracted from PC9 gefitinib-resistant (PC9 GR) cells after treatment with a demethylating agent, 5-azacytidine. Quantitative RT-PCR confirmed the re-expression of KL and S100P after treatment.

including differentiation, imprinting, and oncogenesis (9). In this study, we sought to clarify whether epigenetics have functional roles in acquired resistance to cancer treatments. We focused on aberrant DNA methylations occurring through acquired resistance to gefitinib, one of the clinically available EGFR-TKIs. Although there are many reports on the relationship between cisplatin sensitivity or resistance and DNA methylation $(19,20)$, only few reports are available regarding the relationship between EGFR-TKI resistance and DNA methylation, and none of them used a comprehensive approach (21-23). In this study, two comprehensive analysis tools, cDNA microarray for gene expression and Infinium assay for DNA methylation, were used. Epigenetic gene regulation is a complex biological process that can change the expression of numerous genes. Thus, we expected that the combination of the two comprehensive analyses could be a powerful tool to appropriately detect a number of subtle, but meaningful, epigenetic changes. As a result, 29 genes, which presented a decreased mRNA expression and an increased DNA methylation level, were identified 
A

KL

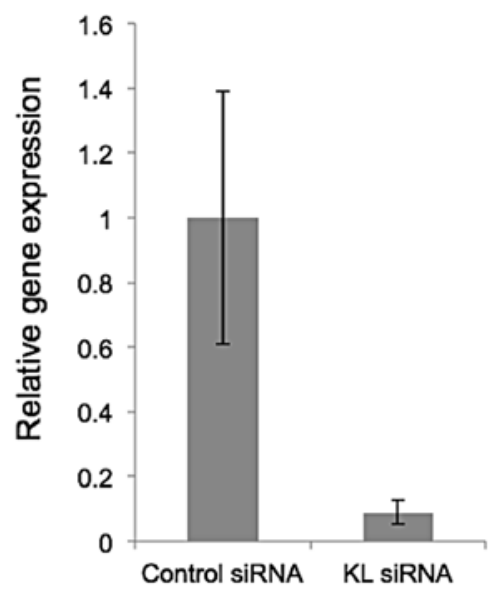

S100P

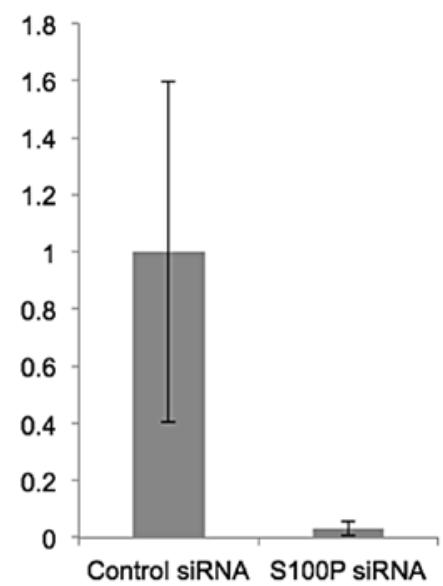

B

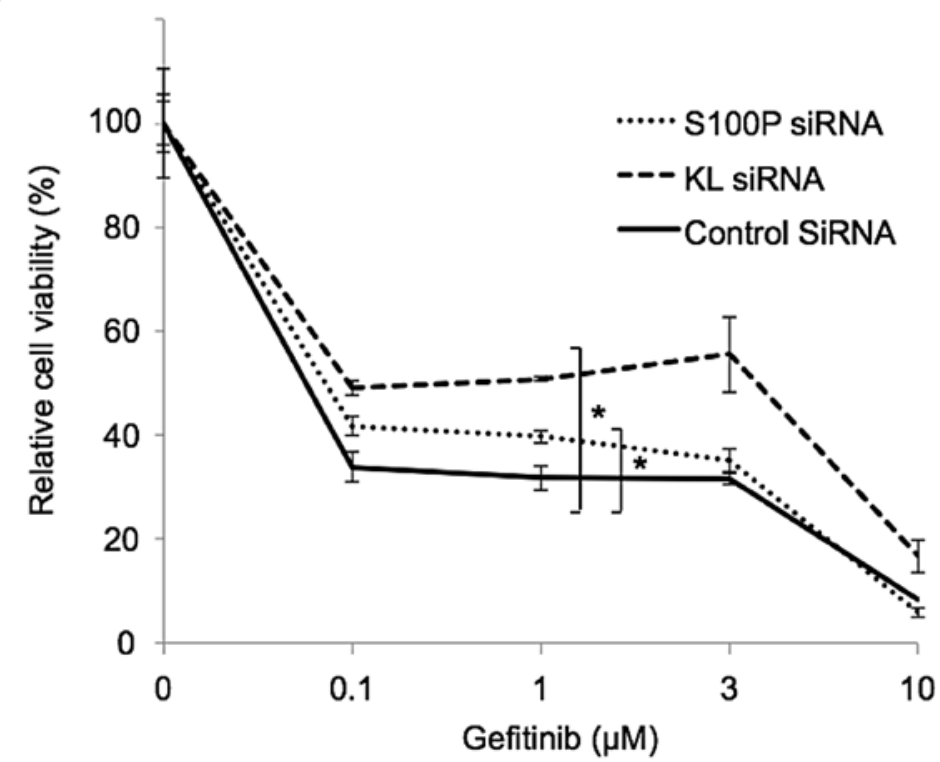

Figure 5. Gefitinib-sensitive PC9 cells transfected with siRNA against $K L$ or S100P. (A) Efficient knockdown of KL (left) and S100P (right) by siRNA confirmed by quantitative reverse transcription-polymerase chain reaction (RT-PCR). (B) MTS proliferation assay results with or without gefitinib are shown. Viability curves of PC9 naïve (PC9 na) cells transfected with siRNA against $K L$, S100P, or the negative control (Control siRNA) and treated with gefitinib at four different test-drug concentrations are presented. Data were normalized to each untreated control, set to $100 \%$. Data represent the mean $\pm \mathrm{SD}$. ${ }^{*} \mathrm{P}<0.05$, Student's t-tests: Control siRNA vs. KL siRNA- or S100P siRNA-transfected PC9 na cells treated with $1 \mu \mathrm{M}$ gefitinib. siRNA-mediated KL or S100P knockdown rendered cells more resistant to gefitinib.

in PC9 GR cells compared to parental PC9 na cells. We speculated that changes in these gene expressions might have some functional roles in acquired resistance to gefitinib. Of these 29 genes, two genes, $K L$ and S100P, were found to be controlled by DNA methylation and have functional roles in acquired gefitinib resistance. Interestingly, the relationship between FGF2-FGFR1 pathway and $K L$ was reported $(22,27)$. Although we were unable to identify a direct relationship between them in this study, decreased $K L$ expression might influence the FGF2-FGFR1 pathway. The contribution of $K L$ and $S 100 P$ in the acquired resistance was relatively small compared to that of the FGF2-FGFR1 pathway (6), but significant. We expect that a collection of 'small' effects on acquired resistance can induce a biologically or clinically meaningful effect.
Further studies aiming at elucidating the mechanisms involved in acquired resistance are warranted because these can help lung cancer patients who suffer from acquired resistance to EGFR-TKI.

\section{Acknowledgements}

The authors thank Ms. Miyuki Yamamoto and Ms. Mikiko Shibuya for their expert technical assistance. This study was supported in part by Grants-in-Aid for Scientific Research on Priority Areas from the Ministry of Education, Culture, Sports, Science, and Technology of Japan (Tokyo, Japan) to K.S. (Grant no. 22590870), H.Y. (Grant no. 25860656), H.T. (Grant no. 24790822, no. 26860616), and K.N. (Grant no. 23501311). 


\section{References}

1. Herbst RS, Heymach JV and Lippman SM: Lung cancer. N Engl J Med 359: 1367-1380, 2008.

2. Schiller JH, Harrington D, Belani CP, Langer C, Sandler A, Krook J, Zhu J, Johnson DH; Eastern Cooperative Oncology Group. Comparison of four chemotherapy regimens for advanced non-small-cell lung cancer. N Engl J Med 346: 92-98, 2002.

3. Mok TS, Wu YL, Thongprasert S, Yang CH, Chu DT, Saijo N, Sunpaweravong P, Han B, Margono B, Ichinose Y, Nishiwaki Y, Ohe Y, Yang JJ, Chewaskulyong B, Jiang H, Duffield EL, Watkins CL, Armour AA and Fukuoka M: Gefitinib or carboplatin-paclitaxel in pulmonary adenocarcinoma. N Engl J Med 361: 947-957, 2009.

4. Paez JG, Jänne PA, Lee JC, Tracy S, Greulich H, Gabriel S, Herman P, Kaye FJ, Lindeman N, Boggon TJ, Naoki K, Sasaki H, Fujii Y,Eck MJ, Sellers WR, Johnson BE and Meyerson M: EGFR mutations in lung cancer: correlation with clinical response to gefitinib therapy. Science 304: 1497-1500, 2004

5. Lynch TJ, Bell DW, Sordella R, Gurubhagavatula S, Okimoto RA, Brannigan BW, Harris PL, Haserlat SM, Supko JG, Haluska FG, Louis DN, Christiani DC, Settleman J and Haber DA: Activating mutations in the epidermal growth factor receptor underlying responsiveness of non-small-cell lung cancer to gefitinib. N Engl J Med 350: 2129-2139, 2004

6. Terai H, Soejima K, Yasuda H, Nakayama S, Hamamoto J, Arai D, Ishioka K, Ohgino K, Ikemura S, Sato T, Yoda S, Satomi R, Naoki $\mathrm{K}$ and Betsuyaku T: Activation of the FGF2-FGFR 1 autocrine pathway: a novel mechanism of acquired resistance to gefitinib in NSCLC. Mol Cancer Res 11: 759-767, 2013.

7. Cheung HH, Lee TL, Rennert OM and Chan WY: DNA methylation of cancer genome. Birth Defects Res C Embryo Today 87: 335-350, 2009.

8. Heller G, Zielinski CC and Zöchbauer-Müller S: Lung cancer: from single-gene methylation to methylome profiling. Cancer Metastasis Rev 29: 95-107, 2010.

9. Wilting RH and Dannenberg JH: Epigenetic mechanisms in tumorigenesis, tumor cell heterogeneity and drug resistance. Drug Resist Updat 15: 21-38, 2012.

10. Esteller M, Garcia-Foncillas J, Andion E, Goodman SN, Hidalgo OF, Vanaclocha V, Baylin SB and Herman JG: Inactivation of the DNA-repair gene $M G M T$ and the clinical response of gliomas to alkylating agents. N Engl J Med 343: 1350-1354, 2000.

11. Nyce JW: Drug-induced DNA hypermethylation: a potential mediator of acquired drug resistance during cancer chemotherapy. Mutat Res 386: 153-161, 1997.

12. Strathdee G, MacKean MJ, Illand M and Brown R: A role for methylation of the hMLH1 promoter in loss of hMLH1 expression and drug resistance in ovarian cancer. Oncogene 18 $2335-2341,1999$

13. Taniguchi T, Tischkowitz M, Ameziane N, Hodgson SV, Mathew CG, Joenje H, Mok, SC and D'Andrea AD: Disruption of the Fanconi anemia-BRCA pathway in cisplatin-sensitive ovarian tumors. Nat Med 9: 568-574, 2003.

14. Shen DW, Su A, Liang XJ, Pai-Panandiker A and Gottesman MM: Reduced expression of small GTPases and hypermethylation of the folate binding protein gene in cisplatin-resistant cells. Br J Cancer 91: 270-276, 2004.
15. Ibanez de Caceres I, Cortes-Sempere M, Moratilla C, Machado-Pinilla R, Rodriguez-Fanjul V, Manguán-García C, Cejas P, López-Ríos F, Paz-Ares L, de CastroCarpeño J, Nistal M, Belda-Iniesta C and Perona R: IGFBP-3 hypermethylation-derived deficiency mediates cisplatin resistance in non-small-cell lung cancer. Oncogene 29: 1681-1690, 2010.

16. Stone A, Valdés-Mora F, Gee JM, Farrow L, McClelland RA, Fiegl H, Dutkowski C, McCloy RA, Sutherland RL, Musgrove EA and Nicholson RI: Tamoxifen-induced epigenetic silencing of oestrogen-regulated genes in anti-hormone resistant breast cancer. PLoS One 7: e40466, 2012.

17. Zeller C, Dai W, Steele NL, Siddiq A, Walley AJ, Wilhelm-Benartzi CS, Rizzo S, van der Zee A, Plumb JA and Brown R: Candidate DNA methylation drivers of acquired cisplatin resistance in ovarian cancer identified by methylome and expression profiling. Oncogene 31: 4567-4576, 2012.

18. Yu W, Jin C, Lou X, Han X, Li L, He Y, Zhang H, Ma K, Zhu J, Cheng L and Lin B: Global analysis of DNA methylation by Methyl-Capture sequencing reveals epigenetic control of cisplatin resistance in ovarian cancer cell. PLoS One 6: e29450, 2011.

19. Chang X, Monitto CL, Demokan S, Kim MS, Chang SS, Zhong X, Califano JA and Sidransky D: Identification of hypermethylated genes associated with cisplatin resistance in human cancers. Cancer Res 70: 2870-2879, 2010.

20. Zhang X, Li W, Li H, Ma Y, He G and Tan G: Genomic methylation profiling combined with gene expression microarray reveals the aberrant methylation mechanism involved in nasopharyngeal carcinoma taxol resistance. Anticancer Drugs 23: 856-864, 2012.

21. Noro R, Gemma A, Miyanaga A, Kosaihira S, Minegishi Y, Nara M, Kokubo Y, Seike M, Kataoka K, Matsuda K, Okano T, Yoshimura A and Kudoh S: PTEN inactivation in lung cancer cells and the effect of its recovery on treatment with epidermal growth factor receptor tyrosine kinase inhibitors. Int J Oncol 31: 1157-1163, 2007.

22. Ogawa T, Liggett TE, Melnikov AA, Monitto CL, Kusuke D, Shiga K, Kobayashi T, Horii A, Chatterjee A, Levenson VV, Koch WM, Sidransky D and Chang X: Methylation of death-associated protein kinase is associated with cetuximab and erlotinib resistance. Cell Cycle 11: 1656-1663, 2012.

23. Zhu J, Wang Y, Duan J, Bai H, Wang Z, Wei L, Zhao J, Zhuo M, Wang S, Yang L, An T, Wu M and Wang J: DNA Methylation status of Wnt antagonist SFRP5 can predict the response to the EGFR-tyrosine kinase inhibitor therapy in non-small cell lung cancer. J Exp Clin Cancer Res 31: 80, 2012.

24. Li XY, Wu JZ, Cao HX, Ma R, Wu JQ, Zhong YJ and Feng JF: Blockade of DNA methylation enhances the therapeutic effect of gefitinib in non-small cell lung cancer cells. Oncol Rep 29: 1975-1982, 2013

25. Costa DB, Halmos B, Kumar A, Schumer ST, Huberman MS Boggon TJ, Tenen DG and Kobayashi S: BIM mediates EGFR tyrosine kinase inhibitor-induced apoptosis in lung cancers with oncogenic EGFR mutations. PLoS Med 4: 1669-1680, 2007.

26. Sharma S, Kelly TK and Jones PA: Epigenetics in cancer. Carcinogenesis 31: 27-36, 2010.

27. Wang Y, Chen L, Huang G, He D, He J, Xu W, Zou C, Zong F, Li Y, Chen B, Wu S, Zhao W and Wu J: Klotho sensitizes human lung cancer cell line to cisplatin via PI3k/Akt pathway. PLoS One 8: e57391, 2013. 